\title{
Arm Posture Tracking Using a Smartwatch
}

\author{
Sheng Shen \\ University of Illinois at Urbana-Champaign \\ sshen19@illinois.edu
}

\begin{abstract}
In this extended abstract, we present our current work in tracking arm postures using only one smartwatch. Our system fuses data from IMU sensors and observations from human kinematics into a hidden Markov model to continuously estimate the 3D arm posture. We hope with some additional work, this could become a useful underlay to a broad class of gesture-based applications. We also discuss potential future works along this direction.
\end{abstract}

\section{Keywords}

Gesture, arm posture, kinematics, anatomy, tracking, smartwatch, hidden Markov model, accelerometer

\section{INTRODUCTION}

Analytics on arm motion has provided valuable information to human activity sensing. Various machine learning algorithms have been applied [2,4] to detect meaningful arm gestures, including smoking, eating, etc., using a smartwatch or a wristband. Some other applications, such as golf swing analysis, require tracking of the full arm posture. Arm posture represents the $3 \mathrm{D}$ geometric model of the arm, and is define by 3 parameters: elbow location, wrist location and wrist rotation (which captures the rotation of the wrist/hand around forearm's pointing direction). Any arm gesture is simply a specific sequence of arm postures that carries semantic meaning. Thus, posture tracking can serve as the underlay to any application-defined gesture recognition.

Today's solutions for tracking arm postures either use computer vision techniques [1] or paste multiple motion sensors on different segments of the arm [3]. We aim to track arm postures using the IMU sensors on a smartwatch alone, without any training (similar to Kinect) [5]. Three main challenges have to be addressed: (1) Arm motion consists of both wrist and elbow motions whereas a smartwatch is a single noisy measuring device on the wrist; (2) The state space of the arm posture is likely to be large, thus efficient state estimation algorithm is required; (3) Unlike in many indoor/outdoor location tracking systems, in

Permission to make digital or hard copies of part or all of this work for personal or classroom use is granted without fee provided that copies are not made or distributed for profit or commercial advantage and that copies bear this notice and the full citation on the first page. Copyrights for third-party components of this work must be honored. For all other uses, contact the owner/author(s).

Ph.D. Forum'16 June 25-30 2016, Singapore, Singapore

(C) 2016 Copyright held by the owner/author(s).

ACM ISBN 978-1-4503-4331-2/16/06.

DOI: http://dx.doi.org/10.1145/2930056.2933324 posture tracking there is hardly any landmark to help calibrate the system and reset the tracking error.

The innovations that we bring are two-fold. First, from the anatomical model of the arm, we observe that for any given wrist orientation, the space of locations where elbow and wrist can reach is limited. We leverage this observation to constrain the search space of arm postures to a small point cloud. Second, we use these point clouds as priors and estimate the translational motion of the elbow inside a hidden Markov model (HMM) framework. Wrist location can be computed as a shift of elbow location along the pointing direction of the forearm, ultimately leading to the tracking of the entire arm.

\section{OPPORTUNITY}

We observe that, due to human arm joint limitation, for any fixed wrist orientation, the space where the elbow and the wrist can possibly reach is small. Figure 1 shows 3 examples corresponding to 3 fixed wrist orientations (denoted as $\mathrm{X}, \mathrm{Y}, \mathrm{Z}$ vectors in $3 \mathrm{D}$ space), in which (light) green and (dark) red point clouds represent possible locations for the wrist and the elbow, respectively. While in general the elbow can move on a 3D sphere (centered at the shoulder and the radius is upper arm length), and the wrist can move within a 3D space (also centered at the shoulder and the radius is full arm length), once the wrist orientation is known, the uncertainty over their locations is much smaller.
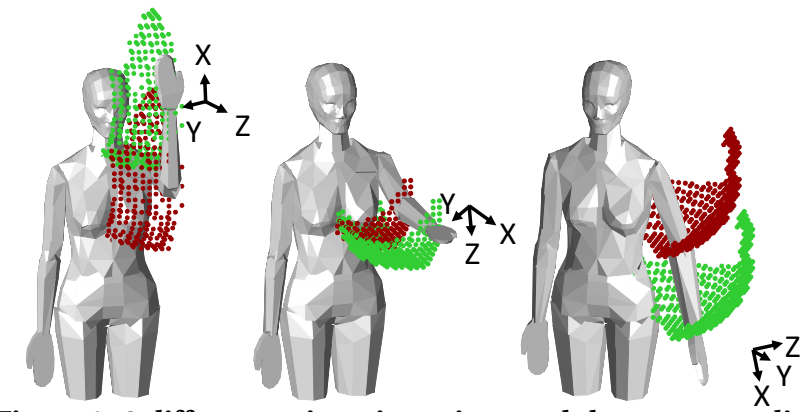

Figure 1: 3 different wrist orientations and the corresponding wrist and elbow point clouds (i.e., possible locations).

To generalize this observation, we looked into human arm kinematics and derived the relationship between wrist orientation and possible wrist/elbow locations. We find that, 5 joint angles, 3 on the shoulder and 2 on the elbow, actually determine the posture of the entire arm, as shown in Figure 2. Assume the lengths of upper arm and forearm are known, then elbow location, wrist location and wrist orientation are all functions of these 5 angles. Also, each joint angle has its own range of motion. 


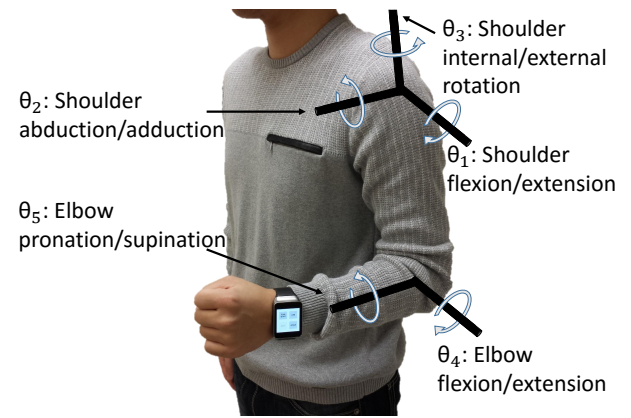

Figure 2: Arm model with 5 rotational joint angles.

Given this arm joint model, we aim to find the elbow/wrist location point clouds that correspond to each wrist orientation. We derive this mapping as follows: for each wrist orientation, we enumerate all possible combinations of 5 angles (within their ranges of motion) that can generate that wrist orientation, and compute the corresponding elbow locations as the elbow point cloud. For any wrist orientation, the wrist point cloud is simply a shift of the elbow point cloud along the forearm's pointing direction (which is the $\mathrm{X}$ direction of wrist orientation). We derive this mapping for all wrist orientations. It turns out in the median case, an elbow point cloud only covers $8.3 \%$ of the area of the entire $3 \mathrm{D}$ sphere that the elbow can move on.

\section{POSTURE INFERENCE}

We borrow the state-of-the-art orientation estimation technique [6] to continuously estimate the watch (same as wrist) orientation from IMU sensors. Given reasonably accurate estimation of wrist orientations (median error $\approx 5^{\circ}$ ) and their mapping to elbow/wrist point clouds, posture tracking problem boils down to elbow location tracking within a sequence of point clouds, i.e., to find the correct elbow point inside each elbow point cloud.

In fact, given that the point clouds are already small, simply computing the average point of each point cloud as the estimation of elbow location might already achieve good accuracy. However, we find much room for improvement. Specifically, we find the accelerometer of the watch can actually be used to infer the motion of the elbow. The accelerometer estimates the (global) wrist acceleration, but added by the constant gravity and projected to the watch's local coordinate system. Given continuous estimation of wrist orientation, we can undo this projection and addition to extract the wrist acceleration alone. Meanwhile, from wrist orientation estimation, we also understand how the forearm rotates relative to the elbow, hence can compute the wristto-elbow relative acceleration. Subtracting this from wrist acceleration, we get a (noisy) estimation of the elbow acceleration.

Since three consecutive locations produce two velocities and hence produce one acceleration, we model this elbow location estimation problem as a third-order hidden Markov model (HMM), where the states are all possible elbow location triples (satisfying the point cloud constraint) and the observations are the inferred elbow acceleration. Viterbi algorithm is used to efficiently decode the underlying elbow location sequence.

We evaluated our posture tracking technique on 8 users and asked them to perform random, free-form, meaningless arm gestures. Figure 3 shows the comparison of tracking accuracy for both elbow and wrist, using the simple averaging (real-time) and HMM (offline) algorithms. By using HMM, the median errors for the $\langle$ elbow, wrist $\rangle$ have reduced from $\langle 12.0 \mathrm{~cm}, 13.3 \mathrm{~cm}\rangle$ to $\langle 7.9 \mathrm{~cm}, 9.2 \mathrm{~cm}\rangle$. We encourage our read- ers to watch the video demonstration of our system here: http://synrg.csl.illinois.edu/posture/.

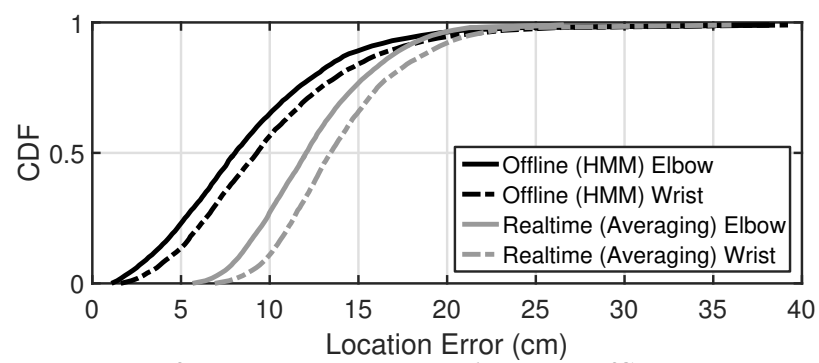

Figure 3: Performance comparison between offline (HMM) and real-time (simple averaging).

\section{CONCLUSION AND FUTURE WORK}

We develop a smartwatch-based arm posture tracking system which leverages human arm kinematics to reduce search space and then uses a hidden Markov model to estimate elbow/wrist location. We believe this is a step forward in posture tracking, and with some more work, could become the building block to many gesture-based applications.

Our broader goal is to track body motion with the minimum number of wearable sensors. As a first step investigation, we proved that tracking arm motion with only one smartwatch is indeed possible. However, our current system assumes the user is standing still. In the future we plan to relax this assumption and track arm motion on the move, possibly by adding another IMU sensor (e.g., a smartphone).

In the long run, we are interested in such a scenario where one can stick several small IMU sensors to different positions of her body and start tracking whole body posture immediately. Two related questions are: (1) What is the least amount of sensors required? (2) What is their best placement? We believe more opportunities will emerge as we look deeper into human skeletal structure, and we aim to investigate these questions over the following years to develop a convenient platform for whole body posture tracking.

\section{REFERENCES}

[1] Microsoft kinect. https://dev.windows.com/en-us/kinect.

[2] Y. Dong, A. Hoover, J. Scisco, and E. Muth. A new method for measuring meal intake in humans via automated wrist motion tracking. Applied psychophysiology and biofeedback, 2012.

[3] M. El-Gohary and J. McNames. Shoulder and elbow joint angle tracking with inertial sensors. Biomedical Engineering, IEEE Transactions on, 2012.

[4] A. Parate, M.-C. Chiu, C. Chadowitz, D. Ganesan, and E. Kalogerakis. Risq: Recognizing smoking gestures with inertial sensors on a wristband. In Proceedings of the 12th annual international conference on Mobile systems, applications, and services. ACM, 2014.

[5] S. Shen, H. Wang, and R. Roy Choudhury. I am a smartwatch and I can track my user's arm. In Proceedings of the 14th annual international conference on Mobile systems, applications, and services. ACM, 2016.

[6] P. Zhou, M. Li, and G. Shen. Use it free: Instantly knowing your phone attitude. In Proceedings of the 20th annual international conference on Mobile computing and networking. ACM, 2014. 